\title{
Correction to: Body fat Indices as Effective Predictors of Insulin Resistance in Obstructive Sleep Apnea: Evidence From a Cross-Sectional and Longitudinal Study
}

\section{BFI as Predictors of IR in OSA}

Ruobing Wei ${ }^{1}$. Zhenfei Gao ${ }^{2}$ Huajun Xu ${ }^{1,3} \cdot$ Cuiping Jiang ${ }^{4} \cdot$ Xinyi Li ${ }^{1} \cdot$ Yupu Liu ${ }^{2}$. Jianyin Zou ${ }^{3} \cdot$ Huaming Zhu $^{1}$. Hongliang $\mathrm{Yi}^{2} \cdot$ Jian Guan ${ }^{1,3} \cdot$ Shankai Yin ${ }^{1}$

Published online: 22 March 2021

(C) Springer Science+Business Media, LLC, part of Springer Nature 2021

\section{Correction to: Obesity Surgery}

https://doi.org/10.1007/s11695-021-05261-9

In the original article, some author affiliations were incorrectly or incompletely assigned. They are correct here.

Publisher's Note Springer Nature remains neutral with regard to jurisdictional claims in published maps and institutional affiliations.

Ruobing Wei and Zhenfei Gao contributed equally to this work.

The online version of the original article can be found at https://doi.org/ 10.1007/s11695-021-05261-9

Huajun $\mathrm{Xu}$

sunnydayxu2010@163.com

$\bowtie$ Cuiping Jiang

litthat@126.com

$\triangle$ Jian Guan

guanjian0606@sina.com

1 Department of Otolaryngology Head and Neck Surgery \& Center of Sleep Medicine, Shanghai Jiao Tong University Affiliated Sixth People's Hospital, Yishan Road 600, Shanghai 200233, China

2 Otolaryngological Institute of Shanghai Jiao Tong University, Yishan Road 600, Shanghai 200233, China

3 Shanghai Key Laboratory of Sleep Disordered Breathing, Yishan Road 600, Shanghai 200233, China

4 Department of Endocrinology, Huadong Hospital Affiliated to Fudan University, Huashan Road 433, Shanghai 200040, Shanghai, China 